\title{
Contractile Analysis with Kriging Based on MR Myocardial Velocity Imaging
}

\author{
Su-Lin Lee, Andrew Huntbatch, and Guang-Zhong Yang \\ Royal Society/Wolfson Foundation MIC Laboratory, \\ Imperial College London, United Kingdom \\ \{su-lin.lee, a.huntbatch,g.z.yang\}@imperial.ac.uk
}

\begin{abstract}
Diagnosis and treatment of coronary artery disease requires a full understanding of the intrinsic contractile mechanics of the heart. MR myocardial velocity imaging is a promising technique for revealing intramural cardiac motion but its ability to depict 3D strain tensor distribution is constrained by anisotropic voxel coverage of velocity imaging due to limited imaging slices and the achievable SNR in patient studies. This paper introduces a novel Kriging estimator for simultaneously improving the tracking and dense inter-slice estimation of the myocardial velocity data. A harmonic embedding technique is employed to determine point correspondence between left ventricle models between subjects, allowing for a statistical shape model to be reconstructed. The use of different semivariograms is investigated for optimal deformation reconstruction. Results from in vivo data demonstrate a marked improvement in tracking myocardial deformation, thus enhancing the potential clinical value of MR myocardial velocity imaging.
\end{abstract}

\section{Introduction}

Coronary artery disease has been the focus of much research due to its considerable morbidity and poor prognosis. The interpretation and prediction of changes induced require a full understanding of the underlying mechanics of coronary flow and myocardial contraction. While visualization of global changes in contractile patterns is possible, examination of local changes in the myocardium requires a more sensitive and quantitative technique. In recent years, Cardiovascular Magnetic Resonance (CMR) has emerged as a versatile technique for non-invasive assessment of intramural motion of the myocardium and has taken a key role in diagnosing myocardial contractile abnormalities.

Historically, the most popular CMR technique for measuring myocardial deformation is MR tagging [1]. More recently, the use of motion tracking with Harmonic Phase (HARP), displacement encoding (DENSE), and myocardial velocity mapping has received increased attention. For myocardial velocity mapping, recent work on pulse sequence design has significantly improved its sensitivity, SNR, and resilience to blood flow artifacts. Detailed, reliable myocardial contractility information is obtained that can be directly used for myocardial modeling. One of the drawbacks of myocardial velocity mapping is that its direct visualization is less intuitive than 
strain/strain rate information as revealed by myocardial tagging, due to the superimposition of global, as well as local myocardial motion. Reliable integration of myocardial velocity information requires physical based 3D modeling combined with correct noise estimation of the velocity data.

Thus far, research into 3D modeling of intramural cardiac motion using velocity imaging has been limited. Current modeling techniques require extensive a priori data, many of which are difficult to obtain on a per subject basis - for example, material properties and fiber orientation. Bergvall et al. [2] used Fourier tracking to examine myocardial deformation. Motion was calculated using an iterative scheme involving regularization to remove the effects of noise. The method was extended by considering data certainty and regularity of the model to improve performance [3]. Masood et al. [4] introduced the virtual tagging framework to derive strain distribution from myocardial velocity data. In virtual tagging, an artificial tag pattern is superimposed onto the velocity data and the subsequent deformation is observed. If the deformation of the virtual tags is correct, the estimated velocity distribution should be identical to the directly measured CMR velocity data in a least-mean-squares sense. Under this framework, a priori information is not required and hence the technique is suitable for subject specific modeling. More recently, the method has been extended by introducing a prediction framework based on kernel-partial least squares regression to predict the distortion of a fine mesh from that of a coarse one [5]. Results from training sets based on a leave-one-out analysis were shown to be promising but the technique would be more effective with training sets from multiple subjects. For this reason, the left ventricles of the subjects must be aligned and mesh correspondence must be found. With correspondence achieved, a model can be built from the resulting training set; such a model could be used for segmentation or as input to the described prediction framework.

Recent work on myocardial velocity data has revealed problems due to anisotropic voxel coverage caused by limited imaging slices and the achievable SNR in patient studies. The purpose of this paper is to introduce a novel Kriging estimator for simultaneously improving the tracking and dense inter-slice estimation of the myocardial velocity data. Kriging is a geostatistical technique to interpolate the value of a random field and it has been used in 3D medical imaging for isosurface generation and tensor estimation and registration [6, 7]. In this paper, we incorporate the Kriging estimator into the virtual tagging framework with surface harmonic embedding such that improved tracking with material correspondence can be achieved. Detailed validation is performed on in vivo MR velocity data sets from five normal subjects.

\section{Methods}

\subsection{Virtual Tagging and the Kriging Estimator}

The concept of virtual tagging was introduced for myocardial contractility analysis for improved strain visualization to avoid the explicit use of tissue properties that are not readily available in vivo. A 3D grid is overlaid onto the velocity image slices and is manipulated based on the surrounding velocity vectors. The cost function $E$ to be minimized is 


$$
E=\sum_{i}\left|\Delta \vec{v}_{i}\right|^{2} \Delta t^{2}+\alpha \sum_{i}\left|\xi_{i}-1\right| S_{i} .
$$

$\Delta v$ is the difference between the estimated and measured velocity vectors, $\Delta t$ is the difference in time between two consecutive timeframes, $\alpha$ is a weighting function, $\xi$ is the change in volume of each element, and $S$ is the surface area of the element. In this paper, we incorporate the ordinary Kriging estimator into the framework to improve the tracking of the myocardial velocity vectors across the cardiac cycle. Kriging is used to interpolate the estimated velocity value at each pixel based on the surrounding mesh nodal values.

Kriging estimation is a modified linear regression technique and is based on the spatial distribution of the samples. The interpolated value is calculated using a neighborhood of the $N$ closest points surrounding the chosen location. The basic Kriging equation is

$$
\hat{Z}(p)=\sum_{i=1}^{N} w_{i} Z_{i}\left(p_{i}\right)
$$

where $\hat{Z}(p)$ is the estimated value at point $p, Z_{i}\left(p_{i}\right)$ are the known regionalized variables at known points $p_{i}$, and $w_{i}$ are the weights. Kriging is considered the best linear unbiased estimator due to the following two conditions:

$$
\begin{aligned}
& \mathrm{E}(\hat{Z}-Z)=0 \\
& \mathrm{E}(\hat{Z}-Z)^{2} \text { is minimum }
\end{aligned}
$$

The system is solved for by using a Laplacian Multiplier $\lambda$ to obtain this system of equations:

$$
\begin{aligned}
& \sum_{j} w_{i} g\left(h_{i j}\right)+\lambda=g\left(h_{i p}\right) \quad(i=1, \ldots, N) \\
& \sum_{j} w_{j}=1
\end{aligned}
$$

Weights $w$ for each point are calculated based on a variogram, half of the variance of the difference between two variables, describing the degree of spatial dependence of the spatial field. A variogram is the expected squared increment of the values between two locations, making it suitable for interpolation. In some applications, a semivariogram (half the variogram) is chosen by fitting a model to empirical data but many now utilize a model semivariogram $g(h)$.

To our knowledge, the choice of model semivariogram has not been studied in medical image applications. In this paper, we compare three model semivariograms to be used within the virtual tagging framework: the exponential semivariogram,

$$
g(h)=c\left(1-\exp \left(\frac{-3 h}{a}\right)\right)
$$

the Gaussian semivariogram, 


$$
g(h)=c\left(1-\exp \left(\frac{-3 h^{2}}{a^{2}}\right)\right)
$$

and the spherical semivariogram.

$$
g(h)=\left\{\begin{array}{cl}
c \cdot\left(1.5\left(\frac{h}{a}\right)-0.5\left(\frac{h}{a}\right)^{3}\right) & \text { if } h \leq a \\
c & \text { otherwise }
\end{array}\right.
$$

where $h$ is the distance between the samples of interest, $c$ is the sill, i.e., the limit of the variogram tending to infinity distances, and $a$ is the range, a distance in which the difference of the variogram from the sill is negligible. While the sill requires an accurate value, the range value can be dispersed.

\subsection{Data Acquisition}

For in vivo validation of the proposed method, five normal subjects were scanned using a gradient-echo phase-contrast protocol $(\mathrm{TR}=53 \mathrm{~ms}, \mathrm{TE}=7.1 \mathrm{~ms}$, in-plane pixel resolution $=1.17 \times 1.17 \mathrm{~mm}, \mathrm{FOV}=30 \times 30 \mathrm{~cm}, \mathrm{VENC}=-15$ to $+15 \mathrm{~cm} / \mathrm{s})$, obtaining a total of 12 to 14 short axis velocity mapping images of the heart with 10-18 timeframes spanning the cardiac cycle on a 1.5T Siemens Sonata MR scanner. The images were restored using a rigid body motion correction and a Total Variational (TV) restoration technique $[8,9]$. In the sequence, a specially designed black-blood RF pulse is applied every other time frame followed by the imaging pulse and a k-space viewsharing scheme is incorporated to reduce the total scan time needed, hence allowing for one reference image and three orthogonally encoded velocity images to be acquired. Free-breathing data acquisition is possible through the use of diaphragmatic navigator echoes and this ensures geometrical and functional consistency of the 3D cine myocardial velocity data.

\subsection{Volumetric Left Ventricle Model}

The left ventricles are first semi-automatically segmented from the reference MR images. Epi- and endocardial surfaces are generated from the slice contours and a volumetric model is built by connecting these surfaces together. However, the points defining these volumes do not correspond across subjects.

To establish correspondence between the two volume models, surface harmonic embedding was applied [10]. With this technique, the epi- and endocardial surfaces are first harmonically mapped to a hollow half sphere. A template of points is then overlaid on the normalised space, providing each surface, regardless of size or subject, with the same number of points defining it. Correspondence is established across two or more subjects through the use of the Minimum Description Length (MDL) criterion. The points are manipulated such that the MDL cost function is minimised, indicating that the model is compact and is unlikely to have spurious modes of variation when Principal Components Analysis (PCA) is applied. After optimisation is complete, the resultant surfaces are reconnected to reform the volumetric mesh. 
Correspondence is only found between the left ventricles at the first timeframe of each subject. The application of virtual tagging ensures that these points are tracked across time and therefore, correspondence will remain.

\section{Results}

In Figure 1, the difference made by the incorporation of the Kriging estimator is visually presented. The use of the Kriging estimator gives a smoother output, especially with the strains, but still tracks the correct movement of the left ventricle. The differences in the virtual tagging due to the use of different semivariograms are shown in Table 1. While all the errors are at a sub-pixel level, use of the Gaussian and spherical semivariograms currently causes slower convergence of the virtual tagging program.

In Figure 2, the strain results are shown from one subject across the cardiac cycle. The results are as expected with the strains following the expected movement in all components. The heart has complex motion that can be broken down into three components - longitudinal, circumferential, and radial. Longitudinally, the heart shortens and lengthens; radially, the heart wall thickens and thins; and circumferentially, there is twisting, leading to contraction and expansion. Component strains are also examined in the left ventricle at each region of the standard AHA 17 segment model [11] in Figure 2. Longitudinal, radial, and circumferential strains are all displayed - in particular, the longitudinal strains are much improved over many existing techniques.

(a)

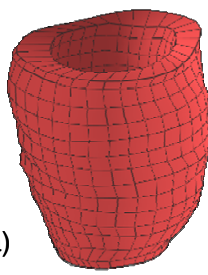

(b)

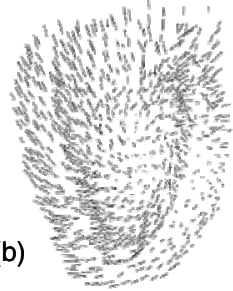

(c)

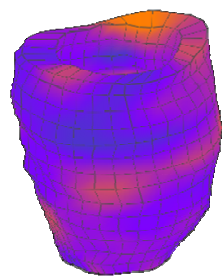

(d)

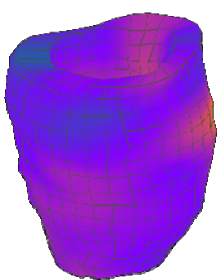

Fig. 1. An example of strain analysis results based on MR phase contrast velocity mapping with and without Kriging. (a) The input shape to the virtual tagging framework, (b) the sampled velocity field showing the noise that can affect the deformation, (c) the deformation found without Kriging, with radial strain overlaid, and (d) the deformation recovered with Kriging.

Table 1. Mean position errors from one subject across three time frames comparing exponential, spherical, and Gaussian model semivariograms

\begin{tabular}{cccc}
\hline \hline & \multicolumn{3}{c}{ Semivariogram } \\
\hline TF & Exponential & Gaussian & Spherical \\
\hline 1 & 0.154 & 0.109 & 0.120 \\
2 & 0.292 & 0.228 & 0.202 \\
3 & 0.166 & 0.192 & 0.120 \\
\hline \hline
\end{tabular}


(a)
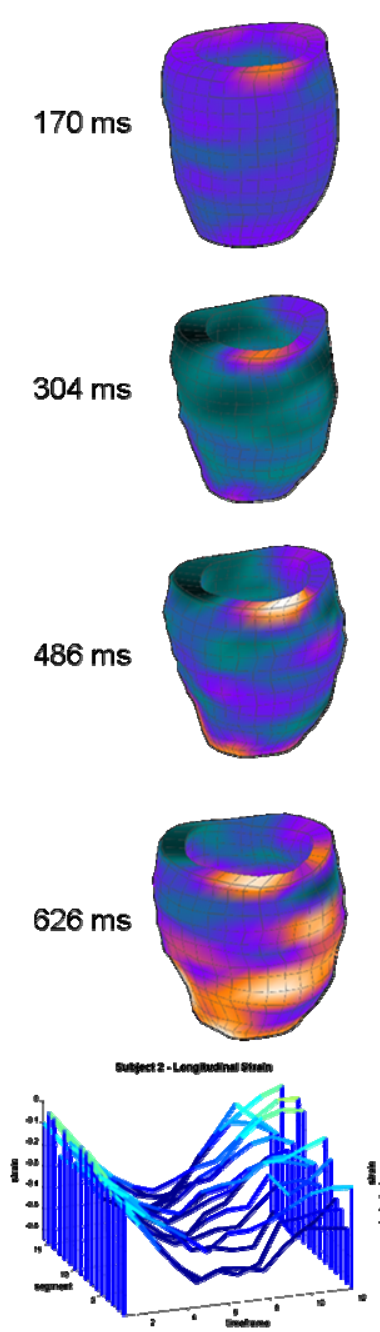

(b)
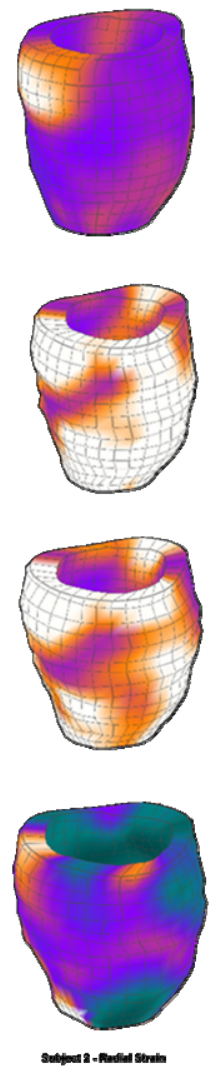

(c)
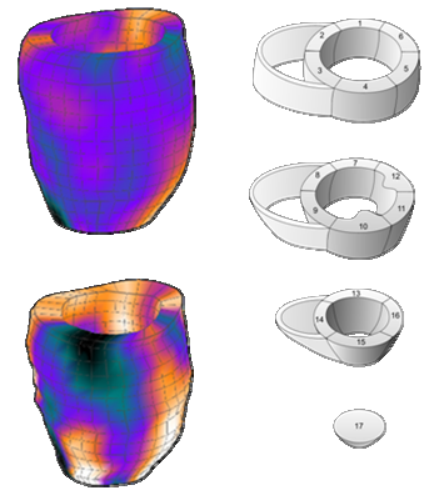
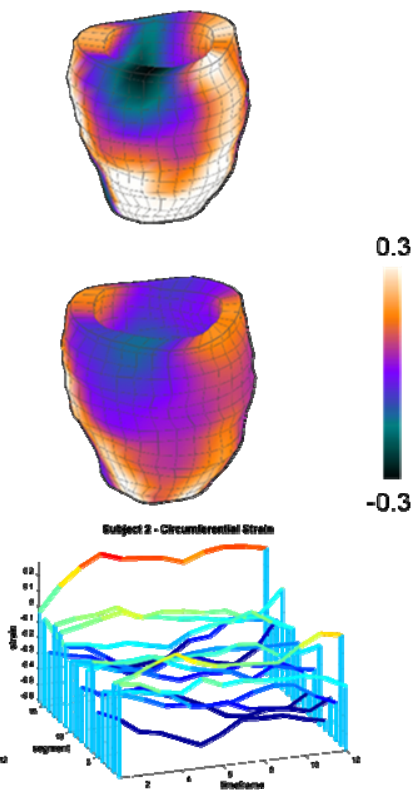

Fig. 2. Strain results from one of the subjects studied - (a) longitudinal, (b) radial, and (c) circumferential strain distribution after Kriging. The seventeen segment model of the left ventricle and the component strains at each of the segment regions are shown to the right and below.

While both the longitudinal strains and radial strains strongly correlate with previous investigations, with the left ventricle clearing showing longitudinal shortening and radial contraction, the circumferential strains are less strong. In Figure 3, the mean values of each strain components are shown for each of the left ventricle segments (except Segment 17 - the apical segment). 

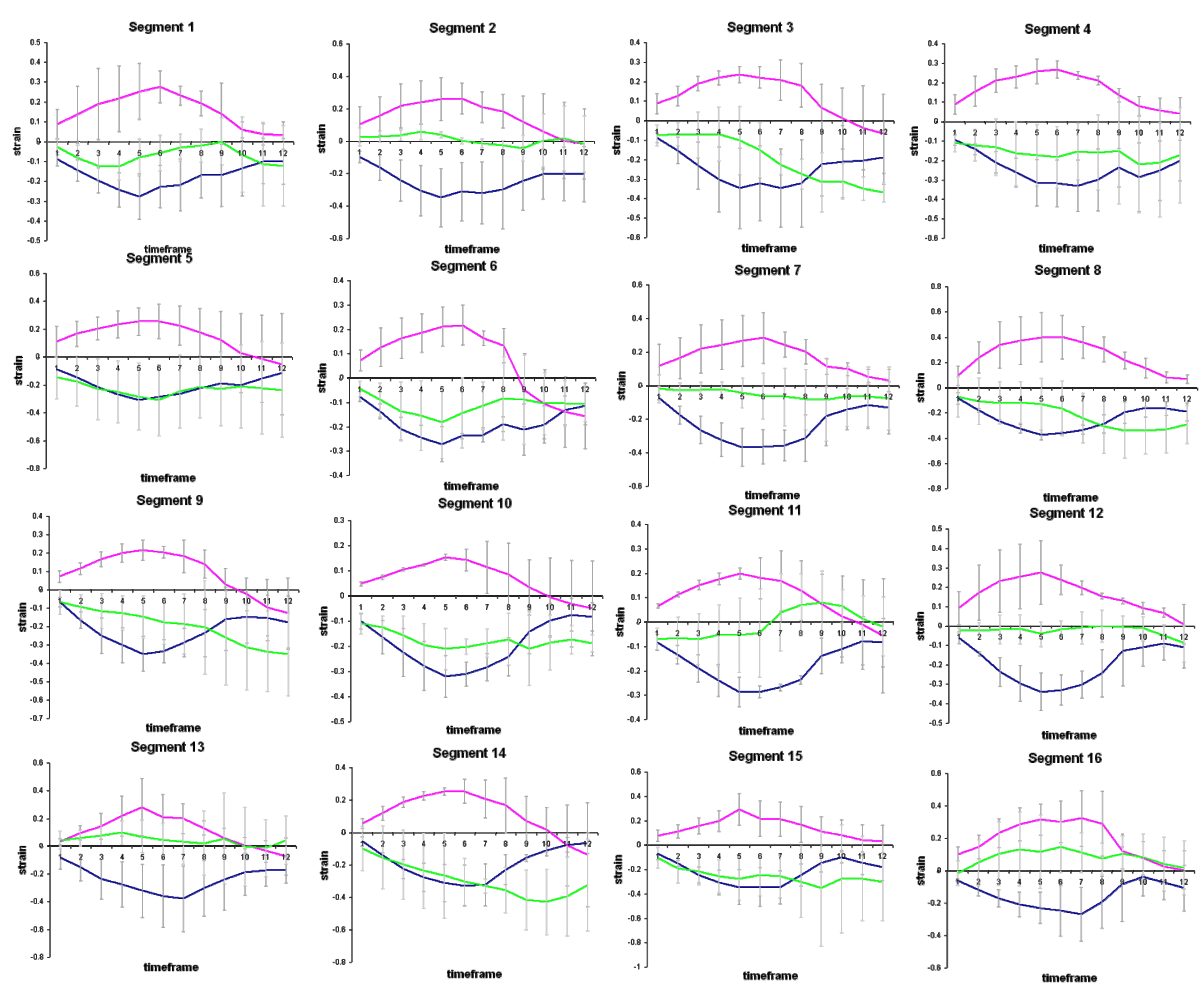

Fig. 3. Mean and standard deviations of the longitudinal (blue), radial (pink), and circumferential (green) strains for each of the segments (except Segment 17) of the standard AHA 17 segment model across all the subjects studied

\section{Conclusions}

In this paper, we have incorporated the Kriging estimator for myocardial strain analysis based on phase contrast velocity mapping. Kriging has the potential for projecting optimal interpolation estimates and is only dependent on the distance to the neighborhood samples. We have shown that the final result is relatively immune to the choice of semivariogram, but the convergence of the algorithm can be affected by different semivariograms. The proposed technique has provided promising results in the tracking of the left ventricle throughout the cardiac cycle with MR velocity images. When combined with the virtual tagging framework, the method allows accurate material correspondence, and thus can serve as a valuable tool for detailed myocardial strain analysis.

\section{Acknowledgements}

The authors wish to thank the team at the Royal Brompton Hospital, London, UK, for their assistance in image acquisition and Robert Merrifield for his assistance with images. 


\section{References}

1. Axel, L., Dougherty, L.: MR Imaging of Motion with Spatial Modulation of Magnetization. Radiology 171, 841-845 (1989)

2. Bergvall, E., Cain, P., Arheden, H., Sparr, G.: A Fast and Highly Automated Approach to Myocardial Motion Analysis Using Phase Contrast Magnetic Resonance Imaging. Journal of Magnetic Resonance Imaging 23, 652-661 (2006)

3. Bergvall, E., Hedstrom, E., Arheden, H., Sparr, G.: Model Based Cardiac Motion Tracking Using Velocity Encoded Magnetic Resonance Imaging. In: Ersbøll, B.K., Pedersen, K.S. (eds.) SCIA 2007. LNCS, vol. 4522, pp. 82-91. Springer, Heidelberg (2007)

4. Masood, S., Gao, J., Yang, G.-Z.: Virtual Tagging: Numerical Considerations and Phantom Validation. IEEE Transactions on Medical Imaging 21, 1123-1131 (2002)

5. Lee, S.-L., Wu, Q., Huntbatch, A., Yang, G.Z.: Predictive K-PLSR Myocardial Contractility Modeling with Phase Contrast MR Velocity Mapping. In: Ayache, N., Ourselin, S., Maeder, A. (eds.) MICCAI 2007, Part II. LNCS, vol. 4792, pp. 866-873. Springer, Heidelberg (2007)

6. Parrott, R.W., Stytz, M.R., Amburn, P., Robinson, D.: Towards Statistically Optimal Interpolation for 3-D Medical Imaging. IEEE Engineering in Medicine and Biology 12, 4959 (1993)

7. Ruiz-Alzola, J., Westin, C.-F., Warfield, S.K., Alberola, C., Maier, S., Kikinis, R.: Nonrigid Registration of 3D Tensor Medical Data. Medical Image Analysis 6, 143-161 (2002)

8. Ng, Y.-H.P., Yang, G.-Z.: Vector-valued image restoration with applications to magnetic resonance velocity imaging. Journal of WSCG 11 (2003)

9. Ng, Y.-H., Carmo, B., Yang, G.-Z.: Flow Field Abstraction and Vortex Detection for MR Velocity Mapping. In: Ellis, R.E., Peters, T.M. (eds.) MICCAI 2003. LNCS, vol. 2878, pp. 424-431. Springer, Heidelberg (2003)

10. Horkaew, P., Yang, G.-Z.: Construction of 3D Dynamic Statistical Deformable Models for Complex Topological Shapes. In: Barillot, C., Haynor, D.R., Hellier, P. (eds.) MICCAI 2004. LNCS, vol. 3216, pp. 217-224. Springer, Heidelberg (2004)

11. Cerqueira, M.D., Weissman, N.J., Dilsizian, V., Jacobs, A.K., Kaul, S., Laskey, W.K., et al.: Standardized Myocardial Segmentation and Nomenclature for Tomographic Imaging of the Heart. Circulation 105, 539-542 (2002) 